\title{
Acknowledgment to Reviewers of Journal of Low Power Electronics and Applications in 2020
}

\author{
Journal of Low Power Electronics and Applications Editorial Office
}

Citation: Journal of Low Power Electronics and Applications Editorial Office. Acknowledgment to Reviewers of Journal of Low Power Electronics and Applications in 2020. J. Low Power Electron. Appl. 2021, 11, 8. https:// doi.org/10.3390/jlpea11010008

Published: 28 January 2021

Publisher's Note: MDPI stays neutral with regard to jurisdictional claims in published maps and institutional affiliations.

Copyright: (C) 2021 by the authors. Licensee MDPI, Basel, Switzerland. This article is an open access article distributed under the terms and conditions of the Creative Commons Attribution (CC BY) license (http://creativecommons.org/licenses /by/4.0/).

MDPI AG, St. Alban-Anlage 66, 4052 Basel, Switzerland

Peer review is the driving force of journal development, and reviewers are gatekeepers who ensure that Journal of Low Power Electronics and Applications maintains its standards for the high quality of its published papers. Thanks to the cooperation of our reviewers, in 2020, the median time to first decision was 18.8 days and the median time to publication was 2.9 days. The editors would like to express their sincere gratitude to the following reviewers for their precious time and dedication, regardless of whether the papers were finally published:

Aiello, Orazio

Aksanli, Baris

Alden, Dochshanov M.

Amsaad, Fathi

Artal-Sevil, Jesús Sergio

Ashraf, Nabil

Bae, Woorham

Basford, Philip J.

Basu, Prabal

Bazzi, Ahmad

Beerel, Peter A.

Belloulata, Kamel

Bertacchini, Alessandro

Bhat, Ganapati

Bogdan, Paul

Bogdan, Razvan

Cagli, Carlo

Cai, Hao

Chakraborty, Koushik

Chang, Yao-Feng

Ciofi, Carmine

Curiac, Daniel-Ioan

De Sousa, Jose T.

Dei, Michele

Dey, Somdip

Dhaou, Imed Ben

Du, Sijun

Dziadak, Bogdan

Elhafyani, Mohamed Larbi

El-Sankary, Kamal

Eshraghian, Jason K.

Ferrante, Alberto
Floros, George

Garcia, Jose Manuel Cano

George, Ioannidis

Georgiou, Orestis

Gericota, Manuel

Gibiino, Gian Piero

Girolami, Marco

Gomez, Ray (Ramon)

Grace, Carl

Graham, David W.

Guachi, Lorena

Guerrero, Esteban Martínez

Gutierrez, Eric

Hasan, Md Sakib

Haubelt, Christian

HEBRARD, Luc

Hoeppner, Sebastian

Hui, Teo Tee

Hwang, Yin-Tsung

Igual, Jorge

Jeloka, Supreet

Jendernalik, Waldemar

Jiang, Hao

Jiménez-Naharro, Raúl

Joyce, Blake

JS, Rajesh

Kakarountas, Athanasios

Karim, Nissar

Khateb, Fabian

Kim, Sungjun

Kim, Youngmin

Kinar, Nicholas J. 
Kirchhoff, Michael

Ko, Hyoung Ho

Kung, Ying-Shieh

Laopoulos, Theodore

Leccese, Fabio

Lee, Huang-Chen

Lee, Sze Sing

Lourenço, Nuno

Madanayake, Arjuna

Manstretta, Danilo

Martínez-García, Herminio

Matsuoka, Toshimasa

Mattia, Oscar

Mehonic, Adnan

Midya, Rivu

Milik, Adam

Miro-Panades, Ivan

Montiel-Nelson, Juan A.

Moon, Jongsub

Munoz-Pacheco, Jesus Manuel

Naik, Ganesh R.

Navickas, Romualdas

Neumann, Philipp

Niotaki, Kyriaki

Pandiev, Ivailo

Park, Jongsun

Passos, Fábio

Pavan, Paolo

Pavlidis, Vasileios

Perri, Stefania

Petrucci, Vinicius

Pietrenko-Dąbrowska, Anna

Portero, Antoni

Prakash, Alok

Psychalinos, Costas

Pudukotai Dinakarrao, Sai Manoj

Qin, Tong

Rahimi, Abbas

Rangarajan, Shriram S.
Ravindran, Arun

Reinbrecht, Cezar

Richelli, Anna

Roohi, Arman

Ruan, Shanq-jang

Săcăleanu, Dragoş Ioan

Sánchez-Solano, Santiago

Savidis, Ioannis

Scotti, Giuseppe

Senhadji-Navarro, Raouf

Serb, Alex

Shayesteh Moghaddam, Narges

Shrivastava, Aatmesh

Silva-Martinez, Jose

Silviu, Folea

Skliarova, Iouliia

Song, Ickhyun

Song, Shuang

Stanchina, William

Sutor, Alexander

Szcześniak, Paweł

Tang, Xiyuan

Thomas, Stewart J.

Tlelo-Cuautle, Esteban

Toledo, Pedro

Tsai, Zuo-Min

Tsiropoulou, Eirini Eleni

Verde, Francesco

Wang, Sen

Wang, Wei

Wildermann, Stefan

$\mathrm{Xi}$, Xiaodan

Xia, Fei

Xu, Qinzhi

Xue, Xiaoyong

Yoon, Hyoseok

Youssef, Ounejjar

Yuan, Bing 